\title{
An Approach to Data Envelopment Analysis Models with Fuzzy Inputs and Outputs by Application of Fuzzy Mathematical Programming
}

\author{
Malekkhayat, $\mathbf{A}^{*}$ \\ *Corresponding Author: Malekkhayat,A
}

\begin{abstract}
Data envelopment analysis (DEA) is a widely used mathematical programming approach for comparing the inputs and outputs by evaluating their relative efficiency. It represents a set of linear programming techniques and uses deterministic data (inputs and outputs), in stable conditions. An efficient integration of production and distribution plans into a unified framework is critical to achieving competitive advantage. In the conventional DEA, all the data assume the form of specific numerical values. However, the observed values of the input and output data in real-life problems are sometimes imprecise or vague. This paper finds efficiency measures with fuzzy inputs and outputs via proposed model. However, a real supply chain operates in a highly dynamic and uncertain environment. From the theory point of view, the objective of this study is to develop a simple and effective Fuzzy DEA model. This paper proposes an interactive evaluation process for measuring the relative efficiencies in fuzzy DEA with consideration of the preferences. it becomes hard for a productivity measurement expert to specify the amount of resources and the outputs as exact scalar numbers. The present paper, applies this method in the application to the oil exploration and production companies of Iran to measure and compare productivity performance of transformation processes when numerical data cannot be specified in exact terms.
\end{abstract}

\section{INTRODUCTION}

Efficiency frontier analysis has been an important approach of evaluating oil exploration and production plant's performance in private and public sectors. There have been many efficiency frontier analysis methods reported in the literature. However, the assumptions made for each of these methods are restrictive. Each of these methodologies has its strength as well as major limitations especially sensitivity of frontier models to data causes to use a fuzzy mathematical programming approach to the assessment of efficiency with DEA models developed.

Any for-profit or non-profit organization requires a set of input resources in order to operate and survive. In return, it provides goods or value-adding services for its clients or stakeholders. The efficiency with which it consumes the resources to provide those services, is measured by the productivity of the organization. The notion of productivity therefore, focuses on exploring the relationship between the results achieved and the resources expended to achieve those results. In its basic form, the productivity is measured by the ratio of outputs (often goods or services) to the input resources (such as labor, capital, management, materials, energy etc).

Two most common measures of productivity are total measure and partial measure. Total measure includes all the input resources used in achieving the desired outputs whereas partial measure focuses on an incomplete list of input factors. If a partial measure focuses on one factor only (e.g. output per labor hour), it is referred to as single factor productivity measure whereas including more than one factor gives multi factor productivity. Sometimes, the use of single factor productivity can be misleading when there is a tradeoff involved among multiple inputs. For example, an organization may procure a better and more expensive software or technology that require less manual processing by its staff. Thus, it is possible to increase labor productivity but at the expense of increased technological costs. Therefore, if an improvement in the single factor productivity has been achieved, it is important to carefully examine the factors responsible for it or alternatively, have a more holistic approach towards productivity

in terms of multi or total productivity measure. The main reasons for process productivity measurement are to monitor and control the organizational performance, judge the effectiveness of our decisions and to create a metric that causes behavioral change among the employees leading towards a productive unit. 
The Data Envelopment Analysis (DEA) approach has been extensively applied in companies to measure the productive efficiency of production entities. Charnes et al. (1978) developed the DEA methodology for measuring relative efficiencies within a group of decision-making units (DMU's) which utilize several inputs to produce a set of outputs. DEA constructs a nonparametric frontier over data points so that all observations lie on or below the frontier. A competing method for computing technical efficiency scores is the stochastic frontier approach (SFA) developed by Aigner et al. (1977) and Meeusen and van den Broeck (1977). DEA approach has been favored over the SFA for several seasons. First, it requires no assumption about the distribution of the underlying data and deviation from the estimated frontier is interpreted purely as inefficiency. Second, it does not require specification of a functional form for the frontier just as economic theory does not imply a particular functional form. Third, multiple inputs and outputs can be considered simultaneously, and fourth, inputs and outputs can be quantified using different units ofmeasurement.

However, DEA requires detailed data about inputs and outputs. It is based on the assumption that all the input and output data are crisp, i.e., all the observations are considered as feasible with probability one, meaning no noise or measurement error is assumed (Simar 2007, Henderson and Zelenyuk 2007). This assumption may not be realistic in production oil companies where inputs and outputs of a decision making unit (DMU) are ever changing because of weather, seasons, operating states and so on (Guo and Tanaka 2001). The dominance of uncertainty in oil production has seen the flourish of studies of production under risk in oil economics (Just and Pope 2001). Factors used in production oil, such as labor, are sometimes difficult to measure in a precise manner. Input measures are often based on accounting data even though the definition of accounting costs differs from that of economic costs by excluding the opportunity cost (Kuosmanen et al., 2007). Producer data may also be available only in linguistic form such as "high yield", "low yield", "labor intensive" or "capital intensive." The convectional DEA approach is very sensitive to data measurement errors and changes in data, including outliers and missing data, can change the efficient frontier significantly. The DEA model is deterministic in nature, meaning that it does not account for statistical noise.

A number of techniques to account for the deterministic nature have beensuggested in the literature, such as the techniques for detecting possible outliers (Cazals et al., 2002) and the stochastic programming approach (Cooper et al., 1998). Notably, Simar and Wilson (1998, 2000a) introduced bootstrapping into the DEA framework to allow for consistent estimation of the production frontier, corresponding efficiency scores, as well asstandard errors and confidence intervals. However, as observed by Kousmanen et al., (2007), the statistical properties and hypothesis tests suggested by Simar and Wilson (2000a, 2000b ) focus exclusively on the effect of the sampling of plants from the production possibilities set and, hence, the bootstrap approach does not allow for data errors of any kind. Therefore, there is need for a model that can adequately represent the stochastic nature of production data at a micro-level.

This paper introduces fuzzy DEA, an approach advanced in the field of mathematical, to measure technical efficiency where data is imprecise. A group of 34 plants producers in iran is used to illustrate how to empirically compute fuzzy technical efficiency scores. The approach incorporates fuzzy set theory and the DEA mathematical programming techniques to compute technical efficiency indices under natural uncertainty inherent in the production processes.

Unlike the convectional DEA model, with a fuzzy DEA model the decision maker can consider different degrees of measurement errors (possibilities) when estimating technical efficiency. Expert judgment expressed in linguistic variables can also be incorporated into the fuzzy DEA models (Guo and Tanaka, 2001).

Fuzzy DEA models are rare in the economics or oil economics literature. A search for "fuzzy DEA" in the oil, AgEcon Search, and EconLit databases returned no items.

Other applications of fuzzy set theory in oil economics include van Kooten et al (2001) who proposed a fuzzy contingent valuation approach to measure uncertain preferences for nonmarket goods. Duval and Featherstone (2002) compared compromise programming and fuzzy programming to a traditional meanvariance approach, and Krcmar and Van Kooten (2008) developed a compromise-fuzzy programming framework to analyze trade-offs of economic development prospects of forest dependent aboriginal communities. 
Analysis of technical efficiency using fuzzy DEA models is very useful to the decision maker and presents several advantages. First, uncertainty in measurement can be incorporated in DEA model at different degrees. Second, linguistic variables can beincorporated into the DEA model, e.g., expert judgment and environmental variables. Third, fuzzy DEA can be used to deal with missing data, and fourth, the decision makercan trace how the efficiency scores vary at different levels of uncertainty. In what follows, the convectional DEA model is presented followed by the basic concepts of fuzzy set theory and how those concepts are integrated into the DEA framework. Then, a literature review of numerical and empirical fuzzy DEA models is presented. The data set is discussed next followed by an application of the fuzzy DEA model to that data and discussion of the results. Then, the article concludes.

\section{LITERATURE REVIEW}

Sengupta (1992) was the first to propose a mathematical programming approach where fuzziness was incorporated into DEA by allowing the objective function and the constraints to be fuzzy. The stochastic DEA model was to be solved using chanceconstrained programming and required the analyst to supply information on expected values of variables, the variance- covariance matrices of all variables, and the probability levels at which the feasibility constraints are to be satisfied. This method was difficult to implement due to those data requirements.

Triantis and Girod (1998) suggested a mathematical programming approach that transforms fuzzy inputs and outputs into crisp data using membership function values. Efficiency scores would then be computed for different membership functions and averaged. Hougaard (1999) suggested an approach that allows the decision maker to include other sources of information such as expert opinion in technical efficiencies computation. Kao and Liu (2000a) suggested the use of -cut level sets to transform fuzzy data into interval data so that the fuzzy model becomes a family of convectional crisp DEA models. This approach was much similar to Guo and Tanaka (2001) who proposed a fuzzy CCR model in which fuzzy constraints, including fuzzy equalities and fuzzy inequalities, were all converted to crisp constraints by predefining different possibility levels.

Lertworasirikul et al (2003) proposed a possibility approach in which fuzzy constraints are treated as fuzzy events and fuzzy DEA model is transformed into possibility DEA model by using possibility measures on fuzzy events. Saati (2002) adopted the -cut level approach, defined the fuzzy CCR model as a possibilityprogramming problem, and transformed it into an interval programming problem. This model could be solved as a crisp LP model and produce crisp efficiency score for each DMU and for each given -cut level. All the above authors used numerical examples to illustrate the application of the proposed fuzzy DEA approach. application of fuzzy DEA models is still in the infancy stage with only one application in oil economics. Hadi-Vencheh and Matin (2011) used an imprecise DEA (IDEA) model to compute the technical efficiency of 15 Iranian wheat producing provinces. Four inputs (acreage, water, wages and number of tractors) and one output (wheat produced) are used. Water and wages are the imprecise variables. The model shows that a DEA model with interval data can be treated as a peculiar DEA model with exact data.

Wu et al. (2006) applied a fuzzy DEA model to determine the efficiency of 24 cross-region bank branches in Canada. The authors incorporating fuzzy environmental variables (income level, population density, and the economy) to assess the performance of bank branches from three different regions: Ontario, Quebec, and Alberta. The assumption made was that different regions may face different external environments that exert significant influence to the performance of different branches. The labels of the environmental variables were linguistic, i.e., "high", "medium", "very good" and "good." The possibility approach and _cut level method as formulated by Lertworasirikul et al.

(2003) was used with a slight modification where both crisp and fuzzy variables are incorporated into the DEA model. The crisp financial input variables used are personnel, equipment, occupancy and other general expenses. Crisp output variables are term deposits, personal loans, small business loans, non term depots and mortgage. The efficiency scores generated by the classical DEA model are compared to those from the Fuzzy DEA model. The study finds that the disadvantage posed by the environment contribute to inefficiency besides the inefficiency that is purely operational. Triantis and Girod (1998) used a threestage approach to measure the technical efficiency performance of one packaging line that is part of a newspaper preprint insertion process. The model has three fuzzy inputs (direct labor, rework and raw materials) and one fuzzy output (packages). In stage one, the vague input and outputs are expressed in 
terms of their risk free and impossible bounds5 and a membership function. In the second stage, the classical DEA models are re-formulated in terms of their risk free and impossible bounds and the membership function for each of the fuzzy input and output variables. The technical efficiency scores are computed in the third stage for different values of the membership function to identify unique sensitive decision making units.

Kao and Liu (2000) applied the concept of fuzzy set theory for representing three missing values in data when studying the efficiencies of 24 university libraries in Taiwan. A triangular membership function is constructed for the missing values by deriving the smallest possible, most possible, and largest possible values from the observed data. Thus, nine libraries end up having fuzzy efficiency scores. The authors observe that interval estimation is more desirable than point estimation of the efficiency score in the absence of certain data. However, they caution that the number of missing data should be restricted to a level such that the number of DMUs, after taking off DMUs with a lot of missing values, should be at least two to three times of the total number of inputs and outputs specified in the model. This study used the ranking approach to rank fuzzy efficiency scores.

\section{Methodology}

Data Envelopment Analysis (DEA) is a methodology based on a Linear Programming (LP) model for evaluating relative efficiencies of Decision Making Units (DMUs) with common inputs and outputs. It is used to ranking and analysis of Decision- Making Units (DMUs), such as industries, universities, hospitals, cities, facilities layout, etc. Data Envelopment Analysis (DEA) is a non-parametric methodology for measuring efficiency within a group of decision- making units (DMUs) that utilize several inputs to produce a set of outputs. DEA models provide efficiency scores that assess the performance of different DMUs in terms of either the use of several inputs or the production of certain outputs. The input-oriented DEA scores vary in $[0,1]$, the unity value indicating the technically efficient units (Leon et al., 2003). The assumption underlying DEA is that all data assume specific numerical values. At first, we use

$\alpha$-cut to convert fuzzy DEA into interval programming as follows Consider $n$ decision-making units, $\mathrm{DMU} j$, where $j=1 \ldots n$. Each DMU consumes input levels $x i j, i=1 \ldots m$, to produce outputs levels $y r j, r$ $=1 \ldots s$. Suppose that $x i j=[x i j \ldots, x m j]^{T}$ and $y r j=T$

$\left[y r j \ldots, y_{s j}\right]$ and are the vectors of inputs and outputs values for DMUj. The relative efficiency score of the DMUo, $o\{1, \ldots, n\}$, is obtained from the following input-oriented DEA model that aims at reducing the input amounts by as much as possible while keeping at least the present output levels:

$$
\operatorname{Min} Z=\theta \text { subject to: } \theta x_{i o} \geq \sum_{j=1}^{n} \lambda_{j} x_{i j}, \forall i, ; y_{r o} \leq \sum_{j=1}^{n} \lambda_{j} y_{r j}, \forall r, ; \lambda_{j} \geq 0
$$

where $\lambda$ indicates the intensity levels which make the activity of each DMU expand or contract to construct a piecewise linear technology (Färe et al. 1994). The DMUo is technically efficient if and only if $\theta=1$, otherwise the DMUo is inefficient. There is an extensive literature on classical DEA models. Cooper et al. (2007) provides a comprehensive review of some of the accomplishments and future prospects of DEA. A major drawback of the DEA model is that the computed relative efficiency scores are very sensitive to noise in data. Any outlier or missing value in the data may cause the efficiency measure of most DMUs to change drastically (Kao and Liu, 2000a; Kao and Liu, 2000b). This makes an approach that is able to deal with inexact numbers, numbers in range or vague measures desirable. Fuzzy set theory can be incorporated in the DEA framework to deal with imprecise data in both the objective function and constraints.

Evaluation of conventional oil plant's performance may be described conveniently within an mathematical framework. In this framework, pertinent inputs are the fuel quantity consumed and installed power, which is the maximum nominal power the plants are initially designed. On the other hand labor inputs contribute to production through control and maintenance services, which also require some capital.

The output is, of course, oil production. But by notice of studies about efficiency measurement of oil generations in Iran which indicate that labor isn't an effective factor Optimization techniques often used in economics are 'crisp' in that a clear distinction is made in a two-valued way between feasible and 
infeasible, and between optimal and nonoptimal solutions (Zimmerman, 1994). The techniques do not allow for gradual transition between these categories, a limitation often referred to as the problem of artificial precision in formalized systems (Geyer-Schulz, 1997). Bellman and Zadeh (1970) were the first to suggest modeling goals and/or constraints in optimization problems as fuzzy sets to account for uncertainty and fuzziness of the decision-making environment.

Fuzzy set theory is a generalization of classical set theory in that the domain of the characteristics function is extended from the discrete set $\{0,1\}$ to the closed real interval $[0,1]$. Zadeh (1965) defined a fuzzy set as a class of objects with continuum grades of membership. Suppose $X$ is a space of objects and $x$ is a generic element of $X$. A fuzzy set, $\tilde{A}$, in $X$ can bedefined as the set of ordered pairs:

$\tilde{A}=\left\{\left(x, u_{A}(x)\right) \mid x \in X\right\}$, (2)where $u A(x): X \rightarrow M$ is the membership function and $M$ is the membership space that varies in the interval $[0,1]$. The closer the value of $u A(x)$ is to one, the greater the membership degree of $X$ to $\tilde{A}$. However, if $M=\{0,1\}$, the set $A$ is non-fuzzy (Triantis and Girod, 1998). A fuzzy set $\tilde{A}$ can be defined precisely by associating with each object $x$ a number between 0 and 1 , which represents its grade of membership in $A$. Thus, $u A(x)=1$ if $x$ is totally in $\mathrm{A}, u A(x)=0$ if $x$ is not in $\mathrm{A}$, and $0<u A(x)<1$ if $x$ is partly in A.

Fuzzy set theory is based on several topological concepts that are beyond the scope of this paper. The interested readers are referred to Kaufmann and Gupta (1991) and Zimmerman (1994) for an introduction to fuzzy sets theory and fuzzy mathematical models. However, terms like fuzzy sets, membership functions and fuzzy numbers will be used several times but no real knowledge of the theory of fuzzy sets is required. Basic concepts relevant to understand this paper are defined:

A set in convectional set theory, $A$, such as a set of large oil plant's $(x)$ that produce at least 1000 litres of oil per day is represented as $\mathrm{A}=\{x \mid \operatorname{oil}(x) \geq 1000\}$. A universal set, $U$, is the set from which all elements are drawn, for example, all oil plant's. The convectional set is defined such that the elements in a universe are divided into two groups: members (those that do belong to it) and non-members (those that do not belong).

A fuzzy number is a quantity whose value is imprecise, rather than exact as is the case with single-valued numbers. Generally, a fuzzy number is a fuzzy subset of a real number, $R$, which is both normal and convex where normal implies that the maximum value of the fuzzy set in $\mathrm{R}$ is 1 . It has a peak or plateau with membership grade 1 , over which the members of the universe are completely in the set. The membership function is increasing towards the peak and decreasing away from it. Fuzzy numbers can be represented as linear, triangular, trapezoidal, or Gaussian.

A triangular fuzzy number, $\tilde{A}$, is a number with piecewise linear membership Functions

$u \tilde{A}(\mathrm{x})$ defined by:

$$
u_{\bar{A}}(x)=\left\{\begin{array}{l}
\mathrm{O}, x<\pi^{\prime} \\
\frac{x-\pi^{\prime}}{\pi^{m}-\pi^{l}}, \pi^{\prime} \leq x \leq \pi^{m}, \\
\frac{\pi^{m}-x}{\pi^{u}-\pi^{m}}, \pi^{m} \leq x \leq \pi^{u}, \\
0, x>\pi^{u}
\end{array}\right.
$$

This can be denoted as a triplet $\left(\pi^{m}, \pi^{l}, \pi^{u}\right)$ where $\pi^{m}, \pi^{l}, \pi^{u}$ are the centre, left spread, and right spread of the number. Figure 1 illustrates an example of a triangular fuzzy number. Letting

$\tilde{A}$ and $\tilde{B}$ to be two triangular fuzzy numbers denoted by ( $a l, a m, a u)$ and $(b l, b m, b u)$, it follows that $\tilde{A} \leq$ $\tilde{B}$ if and only if $a l \leq b l, a m \leq b m$, and $a u \leq b u$.

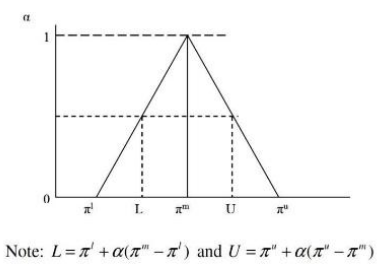

Figure1. A triangular fuzzy number 
The $\alpha$-cut level of a fuzzy set is a crisp subset of $X$ that contains all the elements of $X$ whose membership grades are greater than or equal to the specified value of $\alpha$. This is denoted by

$\tilde{A}_{\alpha}=\left\{\left(x, u_{A}(x)\right) \geq \alpha \mid x \in \mathrm{X}\right\}$. Each $\alpha$-cut level of a fuzzy number is a closed interval which can be represented as $[L(a), U(a)]$, where $L(\alpha)$ is lower bound and $U(\alpha)$ is upper bound at a defined $\alpha$-cut level, $\alpha$. A family of $\alpha$-cut levels determines a fuzzy number. Therefore, the interval of confidence at a given $\alpha$-cut level, where $L$ is lower bound and $U$ is upper bound, can be characterized as :

$\forall \alpha \in[0: 1], A_{\alpha}=\left[L=\alpha\left(\pi^{m}-\pi^{l}\right)+\pi^{l}, U=\pi^{u}-\alpha\left(\pi^{u}-\pi^{m}\right)\right]$.

Fuzzy DEA with Triangular Membership Functions developed a method to find the membership function of the efficiency scores when the observations are fuzzy numbers based on the idea of the $\alpha$-cut level and Zadeh's extension principle. The main idea is to transform the levels of inputs and outputs such that the data lie within bounded intervals, where $L$ and $U$ represent the lower and upper bounds, respectively. Therefore, equation can be reformulated, taking into consideration the fuzzy data, as:

$$
\operatorname{Min} Z=\tilde{\theta} \text { s.t }: \tilde{\theta} \tilde{x}_{i o} \geq \sum_{j=1}^{n} \lambda_{j} \tilde{x}_{i j}, \forall i, ; \tilde{y}_{r o} \leq \sum_{j=1}^{n} \lambda_{j} \tilde{y}_{r j}, \forall r, ; \lambda_{j} \geq 0
$$

The above model can be expanded to indicate the center, lower, and upper bound values as follows:

$\operatorname{Min} Z=\tilde{\theta}$ s.t :

$$
\begin{aligned}
& \left(\tilde{\theta} x_{i o}^{m}, \tilde{\theta} x_{i o}^{l}, \tilde{\theta} x_{i o}^{u}\right) \geq\left(\sum_{j=1}^{n} \lambda_{j} x_{i j}^{m}, \sum_{j=1}^{n} \lambda_{j} x_{i j}^{l}, \sum_{j=1}^{n} \lambda_{j} x_{i j}^{u}\right) \forall i, \\
& \left(y_{r o}^{m}, y_{r o}^{l}, y_{r o}^{u}\right) \leq\left(\sum_{j=1}^{n} \lambda_{j} y_{r j}^{m}, \sum_{j=1}^{n} \lambda_{j} y_{r j}^{l}, \sum_{j=1}^{n} \lambda_{j} y_{r j}^{u}\right) \forall r, \\
& \lambda_{j} \geq 0
\end{aligned}
$$

This model is fuzzy and the usual linear programming method cannot solve it without being defuzzified. The $\alpha$-cut level and extension principle is used to defuzzify the model by transforming the fuzzy triangular numbers to 'crisp' intervals that are solvable as a series of conventional DEA models as follows:

$$
\begin{aligned}
& \text { Min } Z=\theta \text { subject to: } \\
& {\left[\theta\left(\alpha x_{i o}^{m}+(1-\alpha) x_{i o}^{l}\right), \theta\left(\alpha x_{i o}^{m}+(1-\alpha) x_{i o}^{u}\right)\right] \geq} \\
& {\left[\sum_{j=1}^{n} \lambda_{j}\left(\alpha x_{i j}^{m}+(1-\alpha) x_{i j}^{l}\right), \sum_{j=1}^{n} \lambda_{j}\left(\alpha x_{i j}^{m}+(1-\alpha) x_{i j}^{u}\right)\right] \forall i,} \\
& {\left[\theta\left(\alpha y_{r o}^{m}+(1-\alpha) y_{r o}^{l}\right), \theta\left(\alpha y_{r o}^{m}+(1-\alpha) y_{r o}^{u}\right)\right] \leq} \\
& {\left[\sum_{j=1}^{n} \lambda_{j}\left(\alpha y_{r j}^{m}+(1-\alpha) y_{r j}^{l}\right), \sum_{j=1}^{n} \lambda_{j}\left(\alpha y_{r j}^{m}+(1-\alpha) y_{r j}^{u}\right)\right] \forall i,} \\
& \lambda_{j} \geq 0
\end{aligned}
$$

The model is solved by means of comparing the left hand side (LHS) and right hand side (RHS) of each equality/inequality constraint. The main advantage of the $\alpha$-cut level approach used in this paper is that it provides flexibility for the analyst to set their own acceptable possibility levels for decision making in evaluating and comparing DMUs. Zadeh (1978) suggested that fuzzy sets can be used as a basis for the theory of possibility similar to the way that measures theory provides the basis for the theory of probability. The fuzzy variable is associated with a possibility distribution is the same manner that a random variable is associated with a probability distribution. Therefore, the computed fuzzy efficiency 
scores are viewed as a fuzzy variable in the range $[0,1]$.

\section{Data}

Fuzzy DEA is applied to compute the technical efficiency scores of 34 plants in iran using the

$\alpha$-cut level approach. The oil producers use three inputs (plant, labor, and wells) to produce two outputs (oil and gas). The data is obtained from data sheets who used the convectional DEA to computed technical efficiencies, assuming that either the data is precise or the relationship between inputs and outputs is deterministic. To illustrate the application of fuzzy DEA, uncertainty is introduce in the data by representing the inputs and outputs as symmetric triangular fuzzy numbers with a fuzzy interval. The input and output data can be represented as pairs consisting of centers and spreads as

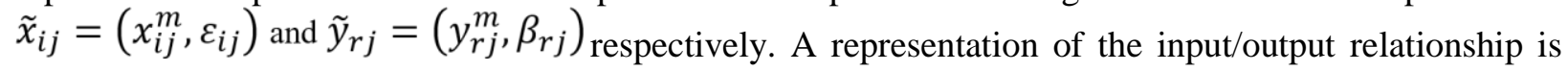
simply:

$\tilde{Y}$ (oil,gas) $=\tilde{X}$ (plant, labor,wells),

where $\tilde{Y}$ and $\tilde{X}$ are matrices of the fuzzy outputs and inputs. The spread for each variable sgenerated as a random number using the random number generator in Microsoft Excel. For the purpose of this study, we assume that the spread for labor is a random number between 0 and

1. The spread for wells is between 1 and 10 and that of plant is between 1 and 20 . The spread of oil is between 100 and 500 and for gas is between 1 and 20. We follow a threestage approach to compute the technical efficiency scores. In the first stage, the inputs and outputs are expressed in terms of symmetric triangular fuzzy numbers and membership functions at six different $\alpha$-cut levels ranging from 0 to 1 . Prespecified intervals of 0.2 are used. In the second stage, the classical DEA model is reformulated as a series of DEA models in-terms of the membership functions for each of the fuzzy input and output variables following equation. In the third stage, fuzzy technical efficiency scores are computed for different membership functions to track how the relative efficiency scores of each plant varies at different possibility levels. The FEAR package in R is used to solve the different LP problems.

\section{RESUlTS}

To demonstrate the validity and practicality of the proposed model, an industrial case inspired from a oil industry is presented. This supply chain network involves three plants, six production lines, three distribution centers located in different customer zones. There are two different types of product groups, and 19 different product types.

The lower bound and upper bound input reducing technical efficiency scores $(\theta \alpha i)$ are presented. The input and output data were assumed to be imprecise and, therefore, the computed efficiency scores are fuzzy too. In general, the lower bound technical efficiency scores $(E i j) L_{\alpha i}$ decreases as the membership function shifts the input and output data from the most precise measurement $(\alpha=1)$ to the most imprecise

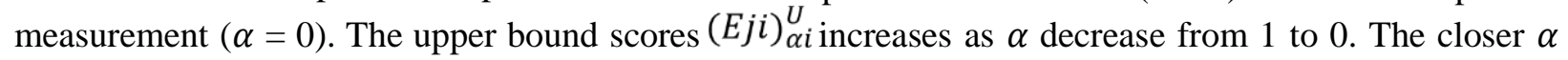
approaches 1 the greater the level of possibility and the lower the degree of uncertainty is. The fuzzy efficiency score lie in a range and the different $\alpha$-cut levels indicate those intervals and the uncertainty level associated with precision in data. Specifically, $\alpha=0$ has the widest interval. On the other hand, the value of $\alpha=1$ is the most likely value of efficiency score.

Using the $\alpha$-cut level approach, the range of a plant's efficiency score at different possibility levels is derived. For example, the efficiency scores for plant 1 at $\alpha$-cut level $=1$ is 0.740 . This deterministic case assumes precision in measurement. At $\alpha$-cut level $=0.8$, the efficiency score range is $[0.737,0.823]$. This indicates that it is possible that the efficiency score of plant 1 will fall between 0.737 and 0.823 at the possibility level 0.8 . The range of the efficiency score at the extremes $(\alpha=0)$ is [0.598, 0.829]. This implies that the efficiency score of plant 1 , relative to other plants, will never exceed 0.829 or fall below 0.598. Results of the other plants at different possibility levels can be interpreted in similar manner. Figure 2 illustrates the membership function of the triangular fuzzy efficiency scores for plant 1 . This represents theextreme range that the frontiers defining the relative technical efficiency scores of each plant are expected to shift due to imprecision in data. The shift of the frontier at $0<\alpha<1$ would fall within this range and would keep on narrowing as $\alpha$ approached 1. 
Realizations of the Solution of the Total Schrödinger Equation from the Link Between Relativity and Quantum Mechanics

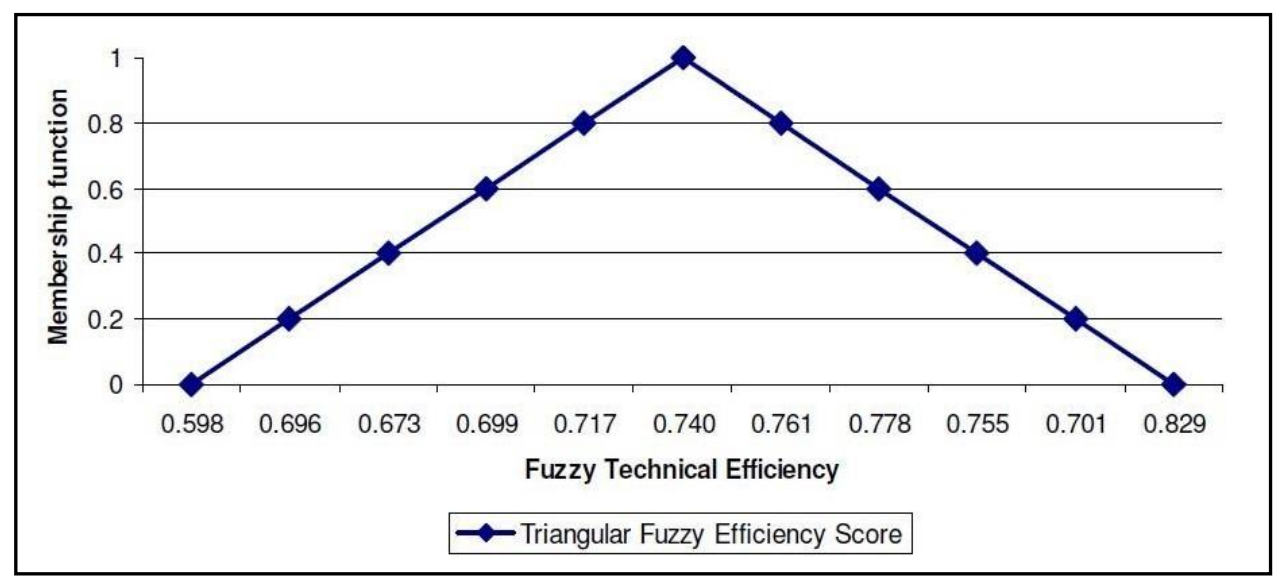

Figure2. Triangular fuzzy efficiency scores for Dairy plant 1

The results from the fuzzy DEA model provide more information to the decision maker compared to the point estimates from the convectional DEA model. The analyst can observe the variation of the technical efficiency profile of each plant from the impossible value when $\alpha$

-cut level $=0$ to the risk-free value when $\alpha$-cut level $=1$. Only four plants, plant 10 , plant 15 , plant 25 and plant 30, remain technical efficient at all $\alpha$-cut levels. Farm 9 becomes technical efficient at the extreme $\alpha$-cut level $=0$. The computed fuzzy efficiency scores need to be ranked in order to determine how each plant performs relative to the other plants in an uncertain environment. The ranking of the fuzzy efficiency scores can be compared to the ranking of scores of the convectional DEA model in order to discriminate which decisionmaking units are sensitive to the variation of the inputs/output variable measurement inaccuracy. We use the Chen and Klein (1997) ranking method to compute an index, I, for ranking fuzzy numbers as:

$$
I_{j}=\frac{\sum_{i=0}^{n}\left(\left(E_{j}\right)_{\alpha i}^{U}-c\right)}{\left[\sum_{i=0}^{n}\left(\left(E_{j}\right)_{\alpha i}^{U}-c\right)-\sum_{i=0}^{n}\left(\left(E_{j}\right)_{\alpha i}^{L}-d\right)\right]}, n \rightarrow \infty,
$$

The lower bound and upper bound efficiency indices are represented by $(E i j) L_{\alpha i}$ and $(E j i) U_{\alpha i \text {. A larger }}$ index indicates the fuzzy number is more preferred. The Chen-Klein's method is used to compute the ranking indices for each plant. The ranking is compared to a ranking of the crisp technical efficiency indices from the classical DEA model and the results are presented in Table

Table1. Ranking of the Crisp and Fuzzy Efficiency Scores

\begin{tabular}{|c|c|c|c|}
\hline & Chen-Klien Index & $\begin{array}{l}\text { CCR } \\
\text { Technical Efficiency }\end{array}$ \\
\hline Rank & DMU & 0.023 & 0.180 \\
\hline $\mathbf{1}$ & plant15 & 0.162 & 0.291 \\
\hline $\mathbf{2}$ & plant 5 & 0.241 & 0.366 \\
\hline $\mathbf{3}$ & plant 33 & 0.277 & 0.393 \\
\hline $\mathbf{4}$ & Plant31 & 0.407 \\
\hline $\mathbf{5}$ & Plant9 & 0.287 & 0.406 \\
\hline $\mathbf{6}$ & Plant23 & 0.290 & 0.465 \\
\hline $\mathbf{7}$ & Plant24 & 0.359 & 0.527 \\
\hline $\mathbf{8}$ & Plant29 & 0.425 & 0.550 \\
\hline $\mathbf{9}$ & Plant32 & 0.450 & 0.548 \\
\hline $\mathbf{1 0}$ & Plant26 & 0.470 & 0.591 \\
\hline $\mathbf{1 1}$ & Plant6 & 0.498 & 0.603 \\
\hline $\mathbf{1 3}$ & Plant12 & 0.517 & 0.620 \\
\hline $\mathbf{1 4}$ & Plant28 & 0.540 & 0.642 \\
\hline $\mathbf{1 5}$ & Plant2 & 0.560 & 0.644 \\
\hline $\mathbf{1 6}$ & Plant17 & 0.562 & 0.665 \\
\hline $\mathbf{1 7}$ & Plant18 & 0.565 & 0.719 \\
\hline $\mathbf{1 8}$ & Plant3 & 0.651 & 0.740 \\
\hline
\end{tabular}


Realizations of the Solution of the Total Schrödinger Equation from the Link Between Relativity and Quantum Mechanics

\begin{tabular}{|c|c|c|c|}
\hline $\mathbf{1 9}$ & Plant27 & 0.665 & 0.735 \\
\hline $\mathbf{2 0}$ & Plant14 & 0.688 & 0.782 \\
\hline $\mathbf{2 1}$ & Plant20 & 0.712 & 0.789 \\
\hline $\mathbf{2 2}$ & Plant34 & 0.731 & 0.779 \\
\hline $\mathbf{2 3}$ & Plant8 & 0.734 & 0.836 \\
\hline $\mathbf{2 4}$ & Plant1 & 0.759 & 0.821 \\
\hline $\mathbf{2 5}$ & Plant19 & 0.795 & 0.855 \\
\hline $\mathbf{2 6}$ & Plant7 & 0.833 & 0.924 \\
\hline $\mathbf{2 7}$ & Plant21 & 0.853 & 0.901 \\
\hline $\mathbf{2 8}$ & Plant13 & 0.916 & 0.938 \\
\hline $\mathbf{2 9}$ & Plant22 & 0.930 & 0.978 \\
\hline $\mathbf{3 0}$ & Plant4 & 0.995 & 1.000 \\
\hline $\mathbf{3 2}$ & Plant16 & 0.999 & 1.000 \\
\hline $\mathbf{3 3}$ & Plant10 & 1.000 & 1.000 \\
\hline $\mathbf{3 4}$ & Plant25 & 1.000 & 1.000 \\
\hline
\end{tabular}

\section{CONCLUSION}

This study propose a model integrating production and distribution plans in a multi-echelon supply chain network with multiple production lines, multiple plants, multiple distribution centers. The model allows the simultaneous determination of assignments of products to production lines, and determination of the quantity of products transported, and determination of the number of vehicles used for each predefined routes. It integrates the strategic decisions concerning product production line assignments with tactical decisions concerning distribution routing.

The main objective of this paper was to introduce fuzzy DEA models by literature review and application as an alternative for analyzing the productive efficiency of oil companies entities in an uncertain environment. Fuzzy DEA models were found to be applicable when expert judgment or environmental variables (linguistic variables) needs be incorporated into the convectional DEA model, when there are missing data and when the measurement of the data is imprecise. An empirical example of symmetrical triangular membership functions was used to illustrate the application of fuzzy DEA to a group of 34 dairy plants in iran. The $\alpha$-cut level approach was used to convert the fuzzy DEA scores into crisp scores. The fuzzy DEA model was able to discriminate the plants whose efficiency performance is sensitive to variation in the inputs/outputs. Compared to the classical DEA model, results from the fuzzy DEA model allow for a determination of robustness and lead to recommendations that are more rigorous.

We conclude by arguing here that it will be interesting to apply empirical fuzzy DEA models in the field of industrial economics using the $\alpha$-cut level approach. Given the incomplete knowledge of input and output measures often used in DEA models, fuzzy DEA models will provide industrial economists with an additional tool for efficiency analysis. Uncertainty always exists in human thinking and judgment. Research in efficiency and productivity analysis should apply recent advancements in DEA that address current concerns. Fuzzy DEA can play an important role for evaluation performance of decision-making units when data are imprecise.

\section{REFERENCES}

[1] Charnes, A., W.W. Cooper., and E. Rhodes. (1978). Measuring the Efficiency of Decision Making Units. European Journal of Operational Research 2(1978):429-444.

[2] Cooper, W.W., Seiford, L.M., Tone, K. and Zhu, J. (2007). Some Models and Measures for Evaluating Performances with DEA: Past Accomplishments and Future Prospects. Journal of Productivity Analysis 28, 151-163.

[3] Geyer-Schulz, A. (1997). Fuzzy Rule-Based Expert Systems and Genetic Machine Learning. PhysicalVerlag, Heidelberg.

[4] Guo, P. and Tanaka, H. (2001). Fuzzy DEA: A Perpetual Evaluation Method. Fuzzy Sets and Systems 119,149-160.

[5] Hougaard, J. L. (1999). Fuzzy Scores of Technical Efficiency. European Journal of Operational Research 115, 529-541.

[6] Krcmar, E., and Van Kooten, G.G. (2008). Economic Development Prospects of Forest- Dependent Communities: Analyzing Trade-offs using a Compromise-fuzzy Programming Framework. American Journal 
of Agricultural Economics 90(4), 1103-1117.

[7] Kuosmanen, T., Post, T. and Scholtes, S. (2007). Non-parametric Tests of Productive Efficiency with Errorsin-Variables. Journal of Econometrics 136, 131-162.

[8] Leon, T., Liern, V., Ruiz, J.L. and Sirvent, I. (2003). A Fuzzy Mathematical Programming Approach to the Assessment of Efficiency with DEA Models. Fuzzy Sets and Systems 139, 407-419.

[9] Lertworasirikul, S., Fang, S., Nuttle, H.L.W. and Joines, J. (2003). Fuzzy BCC Model for Data Envelopment Analysis. Fuzzy Optimization and Decision Making 2, 337- 358.

[10] Peidro, D., Mula, J., Diaz-Madronero, M., \& Vicens, E. (2010). Mathematical programming models for supply chain production and transport planning. European Journal of Operational Research, in press, doi:10.1016/j.ejor.2009.09.008.

[11] Saati, S.M., Memariani, A. and Jahanshahloo, G.R. (2002). Efficiency Analysis and Ranking of DMUs with Fuzzy Data. Fuzzy Optimization and Decision Making 1, 255-267.

[12] Sengupta, J. K. (1992). A Fuzzy System Approach in Data Envelopment Analysis.” Computes Mathematics Application 28, 256-266.

[13] Simar, L. (2007). How to Improve the Performances of DEA/FDH Estimators in the Presence of Noise Journal of Productivity Analysis 28, 183-201.

[14] Strokes, J.R., Tozer, P.R. and Hyde, J. (2007). Identifying Efficient Dairy Producers Using Data Envelopment Analysis. Journal of Dairy Science 90, 2555-2562.

[15] Torabi, S. A., \& Hassani, E. (2013). Multi-site production planning integrating procurement and distribution plans in multi-echelon supply chains: An interactive fuzzy goal programming approach. International Journal of Production Research, 1-25.

[16] Wang, J., \& Shu, Y. F. (2007). A possibilistic decision model for new product supply chain design. European Journal of Operational Research, 177, 1044-1061. Werner, B. (1987). An interactive fuzzy programming system. Fuzzy Sets and Systems, 23, 131-147.

[17] Wu, D., Yang, Z. and Liang, L. (2006). Efficiency Analysis of Cross-Region Bank Branches using Fuzzy Data Envelopment Analysis. Applied Mathematics and Computation 181, 271-281.

[18] Zimmerman, H. J. (1994). Fuzzy Set Theory and Its Applications. 3rd edn. Kluwer- Nijhoff: Boston, USA.

[19] Zadeh, L. A. (1978). Fuzzy Sets as a basis for a Theory of Possibility. Fuzzy Sets and Systems 1(1), 3 - 28.

Citation: Malekkhayat,A, An Approach to Data Envelopment Analysis Models with Fuzzy Inputs and Outputs by Application of Fuzzy Mathematical Programming, International Journal of Scientific and Innovative Mathematical Research (IJSIMR), vol. 8, no. 6, pp. 11-20, 2020. Available : DOI: https://doi.org/ 10.20431/2347-3142.0806003

Copyright: (C) 2020 Authors. This is an open-access article distributed under the terms of the Creative Commons Attribution License, which permits unrestricted use, distribution, and reproduction in any medium, provided the original author and source are credited. 